\title{
Retraso Estatural en Adolescentes de Clase Media Baja
}

\author{
Santiago Muzzo B. ${ }^{1}$ : Raquel Burrows A, ${ }^{2}$; Hernán García B, ${ }^{3}$ y Teobaldo González $Z^{3}$
}

Growth Failure in Adolescents of Middle and Low Socioeconomic Level

\begin{abstract}
A high proportion (45\%) of growth falures was found in an adolescent population of middle and low socio-economic level. Growth failure correlated $(p<0.05)$ with birth weight, learning disabilities and present nutritional status of the adolescents, and also with age at menarche and school level attained by their mothers.

Adolescent females with previous pregnancies, evahuated post partum, had higher growth deficits than non pregnants. There was no correlation between heights of the adolescents mothers and weights of their newborn children but there was a positive one with the baby's heights.
\end{abstract}

El crecimiento estatural del nifio depende de factores genéticos, los cuales se expresan normalmente si el medio ambiente que rodea al sujeto es propicio y capaz de proporcionarle todos los elementos necesarios para un crecimiento normal.

1 Unidad de Endocrinología, Instituto de Nutrición y Tecnología de los Alimentos, Universidad de Chile.

2 Depto. de Pediatría, Universidad Católica de Chile.

3 Servicio de Pediatría, Hospital Sótero del Rio.
Se sabe que factores ambientales, como la nutrición, salud, cultura y estimulación psíquica son de gran importancia para un crecimiento normal. En la medida que estos factores sean adecuados el potencial genético pođrá expresarse totalmente. Muchos autores han demostrado la importancia del factor nutricional en el crecimien. to estatural y asi se ha visto, que los hijos de Japoneses emigrados a U.S.A., tenían igual estatura que el norteamericano promedio, mientras que 
el promedio de estatura en Japón estaba por debajo del promedio en los Estados Unidos ${ }^{1}$.

En nuestro país, se demostró que los niños de clase baja tienen un déficit de $10 \mathrm{cms}$. de estatura a los 5 antos de edad, al compararlos con niños de clase media alta chilena, en tanto que este último tiene estatura similar al promedio norteamericano $^{2}$. En lactantes, preescolares y escolares de clase baja con desnutrición calórico proteica primaria se ha encontrado un gran porcentaje de retraso estatural $^{2-3}$

Todo esto sugiere que el déficit estatural en países sub-desarrollados es consecuencia de la alta prevalencia de desnutrición infantil en clase baja y este déficit es mayor mientras más severa, precoz y duradera haya sido la desnutrición infantil. Por lo tanto, la estatura de un país en desarrollo reflejaría el nivel socioeconómico y nutricional de una población.

Muzzo y cols. ${ }^{4}$ han demostrado que un déficit nutricional en la vida postnatal temprana, en la rata, deja úteros más chicos, con menos células y menor número de receptores de estradiol, irrecuperable a pesar de una rehabilitación nutricional. Esto indica que la desnutrición postnatal precoz en la rata deja un daño uterino irrecuperable, que podria significar una capacidad funcional disminuida y facilitar una desnutrición intrauterina en un embarazo futuro. A pesar de las dificultades para extrapolar los resultados de animales de experimentación al ser humano, ellos sugerirían que las embarazadas de nivel socioeconómico bajo y de talla baja, que posiblemente fueron desnutrjdas precozmente, podrian ser un grupo de alto riesgo de desnutrición intrauterina para sus vástagos.

Nos interesó conocer las caracteristicas del cre. cimiento estatural de un grupo de adolescentes de clase medio baja y correlacionarla con parámetros nutricionales, socioculturales, y otros que influyen en el crecimiento estatural.

\section{MATERIAL Y METQDOS}

En un corte transversal se estudiaron las caracteristicas antropométricas, nutricionales y puberales de 354 adolescentes de ambos sexos que fueron atendidos por diferentes patologías en el Centro de Adolescencia del Consultorio Alejandro del Río. Sus edades fluctuaron entre los 9 y los 18 años. Este grupo de adolescentes incluyó 64 puérperas.

$\mathrm{Se}$ estudió el nivel socioeconómico, estado nutritivo, estatura, desarrollo puberal y realizó una encuesta que indagaba sobre peso de nacimiento, antecedentes mórbidos personales y familiares $\mathrm{y}$ otros que se relacionan con el crecimiento pondoestatural.
El nivel socioeconómico se evaluó según el método de Graffar modificado que comprende los indices de escolaridad, actividad y vivienda del jefe de hogar ${ }^{5}$. El estado nutritivo y la estatura fueron evaluados por los parámetros peso para la edad, peso para la talla y talla para la edad, de acuerdo a las Tablas del National Center of Health Statistics de los Estados Unjoos de Norteanérica (NCHS) ${ }^{6}$.

Se consideró obeso al adolescente cuyo peso para la edad estaba sobre el percentil 90 y el peso para la talla superior al $120 \%$, sobrepeso cuando el peso para la edad estaba entre percentiles 75 y 90 y el peso para la talla entre 110 y $120 \%$, eutrófico si peso/edad estaba entre 25 y 75 y peso/talla entre e] 90 y $110 \%$. Desnutrición si el peso para la edad estaba bajo el percentil 25 y el peso para la talla bajo el $90 \%$. Esto incluía desnutriciones de diferentes grados.

Para estudiar la estatura se separaron los adolescentes con tallas sobre el percentil 90 para la edad; entre los percentiles 10 y 90; entre los percentiles 10 y 5 y bajo el percentil 5 para la edad.

La evaluación del desarrollo puberal se efectuó de acuerdo a la clasificación de las etapas de Tanner?.

El significado de los resultados obtenidos se evaluó estadísticamente según las pruebas de Chi cuadrado y Fischer ${ }^{8}$

\section{RESULTADOS}

El $97 \%$ de los adolescentes estudiados se encontraba en los Graffar 3, 4, 5 y 6 es decir, provenían de un nivel socioeconómico medio y bajo (Tabla 1). En esta y en las siguientes tablas se eliminaron los adolescentes que no tenían la información completa.

Tabla 1.

Nivel socioeconómico (Graffar Modificado) de Adolescentes

$\begin{array}{lrr} & \mathrm{N} & \% \\ \text { Clasc alta }(1-2) & 8 & 2.5 \\ \text { Clase media }(3-4) & 239 & 76.8 \\ \text { Clase baja }(5-6) & 64 & 20.7\end{array}$

En la Tabla 2 se observa que había un $45 \%$ de retraso de talla para la edad, siendo de mayor severidad en las mujeres, pero sin existir diferencias significativas entre ambos sexos. No se encontraron diferencias significativas en la talla entre los niveles medio y bajo. 
Tabla 2

Calidad de la estatura (Talla/Edad) de adolescentes según edad.

\begin{tabular}{lrrrrrrr} 
& \multicolumn{3}{c}{ MUJERES } & \multicolumn{3}{c}{ HOMBRES } & \multicolumn{2}{c}{ TOTAL } \\
& $\mathrm{N}$ & $\%$ & $\mathrm{~N}$ & $\%$ & \multicolumn{1}{c}{$\mathrm{N}$} & $\%$ \\
Sobre & & & & & & \\
percentil 90 & 3 & 1.4 & 0 & 0.0 & 3 & 0.9 \\
Percentil 10-90 & 115 & 52.0 & 71 & 55.0 & 186 & 53.1 \\
Percentil 10-5 & 34 & 15.4 & 24 & 18.6 & 58 & 16.6 \\
Bajo percentil 5 & 69 & 31.2 & 34 & 26.4 & 103 & 29.4 \\
& 221 & 100.0 & 129 & 100.0 & 350 & 100.0
\end{tabular}

Se encontraron relaciones positivas significativas entre la talla actual del adolescente con su peso de nacimiento, escolaridad de la madre, escolaridad del adolescente y trastornos de aprendizaje y relaciones negativas significativas entre talla actual del adolescente con edad de menarquia de la madre y enuresis (Tabla 3).

Tabla 3.

Correlaciones de la estatura actual del adolescente

$\begin{array}{lll} & \mathrm{x}^{2^{(\mathrm{a})}} & \mathrm{p}^{(\mathrm{b})} \\ \text { Peso de nacimiento adolescente } & +3.99<0.05 \\ \text { Escolaridad del adolescente } & +5.10<0.05 \\ \text { Trastornos aprendizaje adolescente } & +3.14<0.05 \\ \text { Antecedentes enuresis adolescente } & -5.20<0.05 \\ \text { Nivel escolar madre del adolescente } & +8.59<0.05 \\ \text { Edad menarquia madre del adolescente } & -7.23<0.05 \\ \text { a }^{2}=\text { Chi cuadrado } & \\ { }_{\mathrm{p}}=\text { Significancia } & \end{array}$

Existió una relación positiva significativa $(p<0.001)$ de la talla con el estado nutritivo expresado como peso/edad (Tabla 4) la cual se hacía negativa $(p<0.01)$ al expresar el estado nutritivo como peso para la talla (Tabla 5 ).

El porcentaje de talla bajo el percentil 10 en el grupo de puérperas adolescentes fue de $58,05 \%$, existiendo un mayor porcentaje de obesidad en las puérperas de talla más baja (Tabla 6). El porcentaje de talla bajo percentil 10 en las embarazadas fue significativamente mayor que en las no emba. razadas $(58.1 \%$ vs. $42.1 \%$ respectivamente, $\mathrm{p}<0.05)$.
Table 4.

Estado nutritivo peso/edad y estatura del adolescente

\begin{tabular}{|c|c|c|c|c|c|c|}
\hline & \multicolumn{6}{|c|}{ ESTATURA (peritentilt) } \\
\hline & \multicolumn{2}{|c|}{90} & \multicolumn{2}{|c|}{$10-90$} & \multicolumn{2}{|c|}{10} \\
\hline & N & $\%$ & N & $\%$ & N & 4 \\
\hline Einceso & 2 & 66.6 & 14 & 7.6 & 5 & 3.2 \\
\hline Eutrofix & $t$ & 33.4 & 99 & 53.8 & 35 & 22.3 \\
\hline \multirow[t]{2}{*}{ Desmutrición } & 0 & 0.0 & וני & 38.6 & 117 & 74.5 \\
\hline & 3 & 100.0 & 184 & 109.0 & 157 & 100.0 \\
\hline$x^{2}=75.3$ & \multicolumn{2}{|c|}{$p<0.001$} & & & & \\
\hline
\end{tabular}

Table 5.

Estado nutritivo peso/talla y estatura del adolescente

\begin{tabular}{|c|c|c|c|c|c|c|}
\hline & \multicolumn{6}{|c|}{ EST ATURA (percentiles) } \\
\hline & \multicolumn{2}{|c|}{90} & \multicolumn{2}{|c|}{$10-90$} & \multicolumn{2}{|c|}{10} \\
\hline & N & $*$ & $N$ & * & N & $\mathbf{4}$ \\
\hline Exceso & 0 & 0.0 & 45 & 24.3 & 62 & 39.5 \\
\hline Eutroiti & 3 & 100.0 & 110 & 59.5 & 86 & 54.8 \\
\hline \multirow[t]{2}{*}{ Dreskestrictán } & Q & 0.0 & 30 & 26.2 & 9 & 5.7 \\
\hline & 3 & 100.0 & 185 & 100.0 & 157 & 100.0 \\
\hline$x^{2}=182$ & $p<$ & & & & & \\
\hline
\end{tabular}

Tabla 6.

Peso al inicio del embarazo y talta en las adolescentes embarazadas

ESTATURA (percentiles) $10-90 \quad 10$

$\begin{array}{lrrrr} & \mathrm{N} & \% & \mathrm{~N} & \% \\ \text { Exceso } & 10 & 43.0 & 18 & 55.0 \\ \text { Normal } & 11 & 48.0 & 10 & 30.0 \\ \text { Déficit } & 2 & 9.0 & 5 & 15.0 \\ & 23 & 100.0 & 33 & 100.0\end{array}$

Si bien no existió una diferencia significativa entre loś pesos de los recién nacidos de madres de talla baja y normal, hubo sin embargo, una correlación significativa positiva entre la talla de la madre y la del recién nacido $(\mathrm{p}<0.05)$.

\section{DISCUSION}

La mayoría de la población de adolescentes estudiada provenía de un nivel socioeconómico medio y bajo. En este grupo había un alto porcentaje de sujetos con talla baja, sin diferencias significativas entre hombres y mujeres, pero con una proporción significativamente mayor en el grupo 
de puérperas adolescentes. La severidad del retraso estatural concuerda con lo encontrado por Calfa A. $y$ cols. ${ }^{9}$ en un grupo de adolescentes puérperas del Gran Santiago de nivel socioeconómico similar. Este mayor porcentaje de retraso en la talla del grupo de adolescentes embarazadas podría, por lo menos en parte, estar condicionado por un déficit nutricional durante el periodo crítico de crecimiento en los primeros afros de vida.

No hubo diferencias significativas en el porcentaje de retraso de talla en los diferentes niveles socioeconómicos del grupo estudiado, los que corresponden en su gran mayoria a nivel medio bajo, bajo y miseria. Esto puede deberse a que en estos niveles los factores ambientales influyen en forma similar.

Se sabe que el bajo peso de nacimiento debido a desnutrición intrauterina de etiología nutricional puede asociarse a una desnutrición postnatal precoz, en cuyo caso se potencia el problema de crecirniento. La relación positiva encontrada entre peso de nacimiento y talla actual, se explicaría porque a mayor déficit nutricional durante el embarazo, menor es el peso de nacimiento y por lo tanto mayot el compromiso estatural.

$\mathrm{La}$ relación positiva entre talla del adolescente y escolaridad de su madre, podría significar que a mejor nivel sociocultural mejor nutrición de la familia, lo que permitirá un mejor estado nutritivo durante la infancia temprana y por lo tanto menor compromiso de la talla en los adolescentes hijos de madres con mayor escolaridad.

Se ha descrito en las mujeres de los niveles socioeconómicos bajos, que la edad de menarquia es más tardia, lo que se explicaría por un estado nutritivo deficiente durante el crecimiento ${ }^{12 \cdot 13}$. Por otro lado existen factores constitucionales que inciden en la edad de menarquia. La relación negativa entre talla actual del adolescente $y$ menarquia de su madre, encontrada en este trabajo, podría hablar en favor de algunos de estos factores.

El hecho de encontrar mejor talia entre los adolescentes que aún estudian y que tienen menos trastornos de aprendizaje, podría traducir un mejor nivel sociocultural y por ende, nutricional.

El hecho de encontrar una relación positiva o negativa entre la talla y el estado nutritivo según se exprese en peso para la edad o peso para la talla respectivamente, se debe al gran porcentaje de retraso de talla existente en esta población. Esto indica la importancia de usar la relación peso para la talla como un mejor índice del estado nutritivo de poblaciones de clase media-baja, donde e] retraso de talla es prevalente.

Se encontró que las adolescentes puérperas con más retardo de talla enfrentaban el embarazo con mayor peso. Esto habla a favor de un posible mecanismo de protección del feto de una eventual desnutrición, ya que el peso de nacimiento de los hijos de madres bajas fue similar al de los hijos de madres de talla normal. Sin embargo, la talla de los recién nacidos se correlacionó en forma positiva con la de la madre, lo que está de acuerdo con lo encontrado por otros autores 10.11 quienes describen que la talla del recién nacido es más parecida a la de la madre que a la del padre. Los resultados permiten concluir que existe un gran porcentaje de adolescentes con retraso de talla en este grupo de bajo nivel socioeconómico, fenóme. no que ya ha sido descrito por otros autores a nivel de escolares y preescolares $\mathbf{1 4 . 1 5}$. Es posible que este retraso estatural se deba a un déficit nutricional durante los primeros años de vida el cual dejó un compromiso de talla que no pudo ser recuperado en su totalidad a pesar de un mejor aporte nutricional posterior. Este fenómeno se ve con mayor frecuencia en la población de adolescentes embarazadas en las que tal vez este déficit nutricional fue más precoz, intenso y prolongado, lo cual podría reflejar un nivel socioeconómico y cultural más bajo que ha condicionado un embarazo más precoz.

\section{RESUMEN}

En un grupo de adolescentes que provenía en su gran mayoría de un nivel socioeconónico medio bajo, bajo y miseria, se encontró un gran porcentaje de sujetos con retraso estatural el que se correlacionó en forma significativa con el peso de nacimiento de ellos, la edad de la menarquia y escolaridad de la madre, trastomos de aprendizaje y estado nutritivo actual del adolescente. Encontramos además que en un subgrupo de adolescentes puérperas, el porcentaje de retraso de talla era mayor que en las no embarazadas y si bien este retraso no tiene influencia en el peso del recién nacido, se relaciona en forma positiva con la talla del recién nacido.

\section{REFERENCIAS}

${ }^{1}$ Grenlich, W."A comparison of the phisical growth and development of American born and native japmeese children". Ann J. Phys. Anthropol. 15: 489, 195 ?.

${ }^{2}$ Barjo, I.; De lo Fuente, M.E.; Ballester, D. Mónckeberg, F.: Donoso, G. Peso y talla de pre-esco lates chilenos urbanos de tres niveles de vida. Rev. Chil. Ped. 36: 525-529, 1965.

3 Burrows, R.; Muzzo, S. Efecto de la estimulación del apetito en la velocidad de recuperación nutricional de la desnutrición infartil. Rev. Chil. Ped. 52: 187-193, 1981. 
${ }^{4}$ Muzzo, S.: Zvaighafi, A. High affinity estrogen receptors in uterus of early malnourished rats. Pediatric. Research. 12: 1030, 1978.

s Alyarez, M,L. Wurgoft, $F$. Mediciones del nivel socioeconómico bajo urbano en madres con lactantes desnutridos. Arch. Latinoamericanos utr. (en prensa).

6 NCHS Growth Curves for Children Birth 18 years. United States. Vital and Health Statistics. Data from the National Health Survey. Series 11 - Number 165.

${ }^{7}$ Marshall, W.A., Tanner, J.M. Arch. Dis, Child. 44: 291 , 1969.

Sidney, $S$. (Editor).. Non parametric statistics for the behavioral sciences. Mc Graw-Hill Book Company, New York, 1956.

9 Calfa, A.L. Importancia de la Nutrición materna como factor de riesgo de retraso del crecimiento intrauterino (RCIU). Tesis para optar al Grado de Magister en Nutrición Humana. U. de Chile, INTA, 1980.

10 Tanner, J.M.; Healey, M.J.R.; Loc Khat, R.D.: Mackennie, J.D. and Whitehouse, R.H. Aberdeen growth study. 1. The prediction of adult body measurements from measurements taken each year form birth to 5 year. Arch. Dis. Child. 31, 372, 1956.

11 Morton. N.E. The inheritance of human birth weight. Ann. Hum. Genet. 20: 125, 1955.

12 Frisch, E.R., Revelle, $R$. Height and weight at menarche and a hypothesis of critical body weights and adolescent events. Science 169: 397, 1970.

13 Lopez, M., Tovar, G., Farid, N., Landaeta, M. y Mendez, $H$. Estudios comparados de la estatura y edad de la menarquía según estrato socjoeconómico en Venezuela. Arch. Latinoamer. Nutr. 31: 740, 1981.

14 Monckeberg, F, Donoso, G., Valiente, S., Arterga, A. Análisis y comentario de la encuesta nutritiva y de las condiciones de vida de la población infantil de la Provincia de Curicó. Rev. Chil. Ped, 38: 522, 1967.

15 Baja, I., Donoso, G., Ballester, D., Monckeberg, F. Peso y talla de preescolares de 3 niveles de vida en la ciudad de Sontiago. Rev. Chil. Ped. 36: S25, 1965. 